\title{
Rapport au savoir musical des professeurs des écoles et pratiques d'enseignement : un transfert parfois complexe
}

To the musical knowledge of teacher's school (the professors of the schools) and teaching practices : a complex transfert

\section{Frédéric Maizières}

\section{OpenEdition Journals}

Édition électronique

URL : http://journals.openedition.org/educationdidactique/1203

DOI : 10.4000/educationdidactique.1203

ISSN : 2111-4838

\section{Éditeur}

Presses universitaires de Rennes

\section{Édition imprimée}

Date de publication : 30 septembre 2011

Pagination : 53-64

ISBN : 978-2-7535-1794-3

ISSN : 1956-3485

\section{Référence électronique}

Frédéric Maizières, « Rapport au savoir musical des professeurs des écoles et pratiques d'enseignement : un transfert parfois complexe », Éducation et didactique [En ligne], 5-2 | 2011, mis en ligne le 30 novembre 2013, consulté le 08 décembre 2020. URL : http://journals.openedition.org/ educationdidactique/1203; DOI : https://doi.org/10.4000/educationdidactique.1203 


\title{
Rapport au savoir musical des professeurs des écoles et pratiques d'enseignement : un transfert parfois complexe
}

\author{
To the musical knowledge of teacher's school (the professors of the schools) and \\ teaching practices : a complex transfert
}

Frédéric Maizières

\section{Introduction}

1 Si les études sur les pratiques enseignantes en Éducation musicale à l'école primaire sont plutôt rares, elles ont toutefois l'avantage de montrer que la musique est présente dans les classes. Et même si son enseignement fait encore souvent figure de "parent pauvre ", des expériences originales existent.

Dans son étude, B. Suchaut (2000) met en évidence des pratiques qui ne sont pas si éloignées des prescriptions, au moins au niveau du temps accordé à la discipline dans la semaine. Ces pratiques sembleraient confirmer l'impact d'un certain nombre d'actions de formation continue et d'expériences pédagogiques dans la région de la Côte-d'Or' ${ }^{1}$.

Mais, d'autres études ou des rapports de l'Inspection Générale confirment que la situation reste inégale et que la musique n'est pas toujours enseignée ou reste très marginale dans certains cas (Baillat, Espinoza, Vincent, 2001 ; MJER, 2003 ; MENESR, 2007). Les principales causes de ces désaffections relèveraient d'un manque de compétences des enseignants, d'où l'idée communément admise que les enseignants qui s'investissent dans les activités musicales avec leurs élèves sont d'abord ceux qui ont bénéficié d'une formation ou qui pratiquent la musique en dehors de leur métier. Le dernier rapport de l'Inspection Générale propose, à ce sujet, de " prendre en compte et de valider sous une forme particulière l'éventuelle formation acquise auparavant » 
(MENESR, 2007, p. 72). Mais il n'existe pas suffisamment de données pour vraiment étayer cette thèse. Et ce n'est sans doute pas un hasard si un certain nombre d'études posent explicitement la question du rapport au savoir musical de l'enseignant pour tenter de comprendre la diversité des pratiques enseignantes en Éducation musicale (Piriot, 1997 ; Suchaut, 2000 ; Jahier, 2006 ; Maizières, 2009).

\section{Une étude récente (Maizières, 2009)}

Une étude menée auprès de 1022 professeurs des écoles de l'académie de Nancy-Metz au cours de l'année 2006 montre que le rapport à la musique de l'enseignant influence peu ses pratiques d'enseignement (Maizières, 2009). Caractérisé comme «l'ensemble des relations qu'un individu entretient avec la musique, compte tenu de ce qui a provoqué ces relations ", le rapport à la musique est étudié sous l'angle des pratiques musicales de l'enseignant au moment de l'enquête, de ses pratiques antérieures, de son éducation (familiale et scolaire), de ses goûts et de son environnement proche. L'étude a donné lieu à diverses analyses statistiques qui ont permis de mettre en évidence certaines corrélations entre le rapport personnel à la musique de l'enseignant et ses pratiques enseignantes en éducation musicale (Maizières, Vilatte, Dupuis, 2007). Toutefois, l'analyse multivariée montre que la part des différences des pratiques d'enseignement expliquée par les variables relatives au rapport à la musique de l'enseignant ne dépasse pas $15 \%$ dans le meilleur des cas. Ce résultat indique donc que $85 \%$ au moins de la diversité des pratiques enseignantes en éducation musicale ne relèverait ni des pratiques musicales personnelles, ni de la formation, ni de l'éducation ou de l'environnement proche de l'enseignant (Maizières, 2009).

5 L'analyse qualitative qui a suivi l'enquête, confirme que ce n'est pas forcément parce que l'enseignant fait de la musique dans sa vie privée ou a bénéficié d'une éducation favorable qu'il met davantage en œuvre des activités musicales dans sa classe, et montre qu'enseigner la musique n'est pas toujours une question de compétences, mais davantage peut-être une question de valeurs. Ces différents résultats indiquent que le transfert entre les propres compétences de l'enseignant et ses pratiques enseignantes demeure complexe.

6 C'est particulièrement la complexité de ce transfert entre le rapport au savoir musical de l'enseignant et ses pratiques enseignantes qui nous préoccupe ici. Il s'agit de comprendre d'une part, comment ce transfert s'effectue, lorsque c'est le cas, et d'autre part, les raisons pour lesquelles il ne s'effectue pas à d'autres moments.

\section{La problématique du rapport au savoir musical}

7 La notion de « rapport au savoir » intéresse les travaux des didacticiens des disciplines dans la mesure où elle se trouve au cœur des processus d'enseignement-apprentissage. Parmi les définitions proposées par B. Charlot, nous retenons que « le rapport au savoir est l'ensemble (organisé) des relations qu'un sujet entretient avec tout ce qui relève de «l'apprendre» et du savoir» (Charlot, 1997, p. 94). Notre analyse se fonde sur le rapport au savoir musical de l'enseignant, c'est-à-dire le fait qu'il y ait un enjeu de savoir dans sa relation avec la musique. L'appropriation correspond à la relation entre le savoir musical et l'enseignant en tant qu'apprenant. La transmission est la relation entre le savoir musical et l'enseignant en tant que formateur. 
8 B. Charlot identifient deux composantes du rapport au savoir : «identitaire " et " épistémique », modulée par une troisième, la dimension "sociale ». Tout rapport au savoir comporte une dimension identitaire dans la mesure où « apprendre fait sens en référence à l'histoire du sujet, à ses attentes, à ses repères, à sa conception de la vie, à ses rapports aux autres, à l'image qu'il a de lui-même et à celle qu'il veut donner aux autres » (Charlot, 1997, p. 84-85). Si le rapport identitaire au savoir correspond à la question "pourquoi apprendre? ", le rapport épistémique au savoir correspond à la question «apprendre, c'est avoir quel type d'activité ?». Le rapport épistémique renvoie à la nature même de l'acte d'apprendre et au fait de savoir. Enfin, le sujet dont on étudie le rapport au savoir existe dans une société qui donne une forme particulière aux dimensions épistémiques et identitaires, c'est l'aspect social du rapport au savoir. Le rapport au savoir est donc le rapport d'un sujet singulier inscrit dans un espace social.

Notre étude pose la question de l'application auprès d'un public d'enseignants des outils socio-linguistiques développés par Escol'2 ${ }^{2}$. Comme nous l'avons souligné ci-dessus, les sujets sont confrontés dans ce cas à deux types d'activités vis-à-vis du savoir, non seulement "apprendre ", mais aussi «enseigner ». Il est donc difficile d'envisager le " rapport au savoir » d'un enseignant sans prendre en compte les deux aspects. Or, même si les membres de l'équipe Escol ont exploré aussi les rapports aux savoirs d'enseignants du primaire et du secondaire, leur théorie rend compte uniquement du rapport à « l'apprendre » et il reste encore à proposer un cadre définissant le rapport à «l'enseigner ». Dans notre cas, le rapport au savoir musical de l'enseignant concerne l'action mise en œuvre pour s' «approprier» le savoir musical, mais aussi pour le "transmettre ". Toutefois, à la lecture des entretiens réalisés dans le cadre de notre étude, nous retrouvons un certain nombre de caractéristiques des processus épistémiques au savoir identifiés par B. Charlot. Pour suivre l'auteur, la posture d'«objectivation-dénomination» suppose un savoir-objet identifiable et «un sujet conscient de s'être approprié un tel savoir » qui pourrait d'ailleurs coïncider avec la forme scolaire du savoir (Charlot, 1997, p. 80). D'autres sujets sont «imbriqués » dans une situation musicale floue, non distanciée, où ce qui leur manque « c'est le rapport à un savoir pensé comme ayant sens et valeur en lui-même, "objectivé » (Charlot, Bautier \& Rochex, 1992, p. 148). Enfin, une troisième posture dominée par « un travail réflexif d'ajustement de la conduite à la situation qui suppose et produit une distanciation vis-à-vis de cette situation, des autres et de soi-même, ainsi qu'une régulation qui peut conduire jusqu'à l'énoncé de règles » (Charlot, 1999, p. 31).

\section{Méthodologie}

10 À la suite de l'enquête menée au cours de l'année 2006 dans l'académie de Nancy-Metz, 16 enseignants ont été interviewés sur leur rapport personnel et professionnel à la musique, notamment leur rapport au savoir musical. Ces 16 enseignants ont été sélectionnés dans l'échantillon de l'enquête à partir d'un certain nombre de critères : leurs pratiques musicales personnelles (solides ou absentes) et leurs pratiques enseignantes en Éducation musicale (solides ou faibles). Il s'est agi, au cours de ces entretiens, de comprendre pourquoi le rapport personnel à la musique des enseignants influençait peu leurs pratiques professionnelles et quelles valeurs mobilisaient en définitive ces enseignants (Maizières, 2009). Les entretiens s'intéressaient aux pratiques 
d'enseignement (temps, activités, objectifs, outils, etc.) et au rapport personnel à la musique des enseignants, notamment leur rapport au savoir musical (pratiques, formation, éducation). Les différentes réponses ont permis de comprendre comment s'était construit leur rapport au savoir musical et quelles composantes du rapport au savoir constituaient pour eux une aide ou au contraire un frein à la mise en œuvre d'activités musicales avec leurs élèves. Précisons que dans cette étude, les enseignants sont désignés par des lettres (de $\mathrm{A}$ à $\mathrm{P}$ ) correspondant uniquement à l'ordre des entretiens. L'enquête antérieure ayant montré le faible poids des critères d'âge, de sexe et d'ancienneté pour expliquer la diversité des pratiques enseignantes en Éducation musicale, nous n'avons pas conservé ces variables dans la sélection. Toutefois, pour respecter une certaine diversité, nous avons interviewé six hommes (A, B, D, H, K, P) et dix femmes (C, E, F, G, I, J, L, M, N, O). Nous avons exclu les débutants (moins de 5 ans d'ancienneté), afin d'entendre des enseignants bien établis dans leur métier. Enfin, l'enquête montrait des différences assez importantes entre le cycle 1 et les classes élémentaires, c'est la raison pour laquelle, en dehors d'un enseignant de maternelle (A en petite section), nous n'avons retenu que des enseignants de cycle 2 (B, C, E, F, I, J, L, P) et de cycle 3 (D, G, H, K, M, N, O).

\section{Quelles pratiques enseignantes pour des professeurs des écoles qui déclarent ne pas faire de musique dans leur vie privée?}

Différentes études montrent que l'écoute de musique représente une activité fréquente dans la population des enseignants du premier degré (Piriot, 1997 ; Maizières, 2009). Les entretiens confirment cette pratique d'écoute, y compris chez les enseignants qui n'ont jamais fait de musique: "Je ne joue pas de musique, mais j'aime bien écouter de la musique» (M). Les goûts sont souvent éclectiques: "J'écoute tout, ça va de Wagner aux Beatles avec une préférence pour Wagner et Jimmy Hendrix » (A), et ne résultent pas toujours d'une recherche esthétique bien objectivée: "J'ai des enfants, donc forcément j'ai des nouveaux airs pour lesquels ils me mettent au courant» (M). Enfin, ces enseignants disent n'avoir bénéficié d'aucune formation ou alors très ponctuellement : " À part en sixième » et en deuxième année d'IUFM «quelques heures comme ça » (G).

Pourtant à l'évocation de leur implication dans les activités musicales avec leurs élèves, ces mêmes enseignants adoptent des postures très différentes que nous présentons successivement. Tout d'abord des enseignants disent faire de la musique avant tout pour respecter les programmes, alors que pour d'autres la musique fait partie des domaines enseignés dans leur classe au même titre que les autres disciplines.

\section{«Simplement histoire de respecter les horaires et d'en faire » (G)}

13 Pour une première catégorie, les pratiques enseignantes se caractérisent par un volume horaire hebdomadaire relativement faible (30 minutes maximum) ${ }^{3}$, sans respecter obligatoirement leur emploi du temps : "Il est 11 heures et quart et en un quart d'heure on n'installe pas quelque chose, donc là on va effectivement écouter de la musique ou faire un chant ou ce genre de choses quoi » (M). Pour ces enseignants, l'éducation musicale s'appuie essentiellement sur le chant "parce que c'est la seule chose que je peux faire faire aux enfants» (M) déclare une enseignante. L'activité reste toutefois limitée dans la plupart 
des cas : «Si j'en pratique deux ou trois, ce sera beaucoup pour moi » (N), précise une autre enseignante à la question du nombre de chants appris en une année scolaire. Et même si l'activité d'écoute est pratiquée quelques fois, elle représente un exercice plus difficile encore: "Ça reste à un petit niveau" $(\mathrm{G})$, "Je ne sais pas dire de qui est le morceau » (E). En dehors d'une découverte par imprégnation, le choix des œuvres ne répond pas toujours à des objectifs précis et l'exploitation reste limitée : "Je leur fais toujours un petit temps d'écoute, j'essaie de leur faire écouter différents registres de musique..., et je leur demande ce qu'ils en pensent, s'ils aiment, s'ils n'aiment pas, à quoi ça leur fait penser... » $(\mathrm{N})$.

14 Toutefois ces enseignants, malgré leurs faibles compétences annoncées et leur petite pratique enseignante déclarée, enregistrent quelques succès : "C'est un plaisir quand la chanson est bien chantée par les enfants, là je me dis : c'est bien, j'ai fait mon travail, j'ai réussi, mon objectif est rempli » (G). Et même si le plaisir est à la fois objectif et gage de réussite, ces enseignants admettent aussi l'importance d'une véritable éducation du goût pour leurs élèves: "Je pense que déjà à l'école on a quand même une mission de faire découvrir des choses assez variées, ils ont besoin de voir des choses pour pouvoir affirmer leur goût » (G).

Et l'on voit bien comment, pour ces enseignants, les activités musicales sont davantage envisagées dans leur classe pour «s'acquitter de [leurs] obligations professionnelles, définies en termes de tâches et de conduites plus qu'en terme d'acquisition de savoirs et de compétences » (Charlot, Bautier \& Rochex, 1992, p. 148). Certes, ces enseignants mesurent l'importance d'une éducation artistique, mais leurs propos montrent qu'ils sont plus ou moins imbriqués dans une confusion entre éducation «à » l'art et éducation " par » l'art : «Je pense que c'est pour se sentir mieux, pour savoir se concentrer, pour s'intéresser à autre chose que leurs petits problèmes... ça leur permet de s'ouvrir un peu et d'appartenir à un groupe... la connaissance d'un patrimoine culturel » (N). On retrouve dans les propos des enseignants de cette première catégorie certaines similitudes avec le processus d'imbrication décrit par B. Charlot. Ils « citent peu de contenus de savoir » et le rapport au savoir est davantage "centré sur les situations, sur l'action, sur les relations, sur la nécessité de "se débrouiller » pour s'adapter... » (Charlot, 1999, p. 31), mais sans véritable distanciation avec l'action elle-même. En dehors du "plaisir » de chanter, ces enseignants peinent à énoncer des objectifs précis et à expliquer leur choix. Il est le plus souvent question de faire découvrir des univers musicaux variés, ou bien leur action se limite à des objectifs très généraux comme l'écoute, l'attention, mais sans véritable démarche didactique. Celle-ci se limite le plus souvent, dans le cas du chant, à une simple imitation du modèle, et dans le cas de l'écoute, à une seule imprégnation, sans véritable analyse de la musique.

\section{" Je fais musique tous les matins parce que c'est une mise en disponibilité du corps et de l'esprit, et de la voix » (J)}

Une deuxième catégorie d'enseignants parmi ceux qui se déclarent sans formation musicale personnelle adopte une posture différente. Malgré leurs connaissances limitées dans certains domaines, comme la musique : « Je ne sais pas jouer de la musique et je n'ai pas de connaissances»(A), ils ne négligent aucun aspect de l'enseignement, notamment celui des arts auquel ils accordent une importance certaine.

17 Dans la classe, la musique occupe un volume horaire assez conséquent (environ une heure hebdomadaire), et ce qui marque la place de cet enseignement, c'est d'abord qu'il 
est fractionné en plusieurs moments, par exemple : «une séance de trois quarts d'heure plus des petits moments par ci par là [pour chanter], si on ne compte pas l'audition... tous les deux jours on écoute un morceau » (0). C'est aussi un enseignement qui «ne passe pas à la trappe » au détriment des autres disciplines. Les deux activités qui caractérisent l'Éducation musicale sont le chant et l'écoute. Pour le chant, les enseignants citent facilement les titres des chansons apprises. Ils expliquent que les difficultés rencontrées peuvent être vaincues par un travail technique régulier : "Un échauffement plus spécifique pour les grandes sections CP..., alors qu'en CE1, je vais plus axer l'échauffement sur la respiration... » (L). Contrairement à la catégorie précédente, l'activité d'écoute s'appuie sur des œuvres clairement identifiées et qui font une large place au répertoire " classique »: "Les concertos brandebourgeois », «les Quatuors de Haydn", "Le Trouvère de Verdi», "la musique du film Les caprices du fleuve», etc., En revanche, les activités instrumentales sont rares par manque de maîtrise: "J'ai un xylophone, je m'en sers mais n'étant pas praticienne du tout, je ne sais vraiment pas m'en servir, alors les enfants viennent éventuellement taper un rythme et puis on reproduit... mais des activités instrumentales, non $»(0)$.

Les propos révèlent que malgré cette absence de compétences instrumentales souvent affirmée, les enseignants mettent en œuvre des activités pédagogiques où prédomine la recherche de stratégies, tant sur le plan organisationnel que matériel, par exemple, le "quart d'heure musical quotidien ». Certains enseignants dont l'implication ne se limite pas à leurs seuls élèves "jonglent » avec des contraintes lourdes : "Ça se fait en deux temps parce que je ne peux pas avoir tout le monde en même temps... je fais les grandes sections $C P$ avec l'IME ${ }^{4}$ dans un premier temps, ça fait quatre-vingt gamins et donc après dans un deuxième temps, je fais les CE1, ça fait une cinquantaine de gamins»(L). Une autre enseignante explique que pour donner une certaine assise à l'activité d'écoute elle compile toutes les œuvres étudiées sur un même support que les élèves peuvent ensuite réécouter chez eux : "Ça fait deux années que je fais des compils d'écoute musicale... » (F). Une autre encore dit s'appuyer sur le répertoire traditionnel pendant tout le premier trimestre parce que, explique-t-elle, «en partant du principe que c'est du bagage qui se travaille en famille..., c'est des chants qu'ils peuvent reprendre à la maison, même si les parents ne connaissent pas la musique » (J).

19 Enfin, ce qui caractérise aussi ces pratiques, c'est qu'elles sont motivées par des finalités précises, à la fois musicales et transversales (mémorisation, attention, traitement de l'information, relation à l'autre, affirmation de soi). Outre le plaisir légitime que tout un chacun attend d'une activité musicale, ces enseignants sont à la recherche d'une qualité d'interprétation: "Arriver à chanter, démarrer en même temps, finir en même temps, toutes ces choses assez simples mais pour pouvoir produire quand même quelque chose de beau ensemble " $(\mathrm{F})$. Les activités musicales visent également l'accès à une culture: "faire répertorier des genres de musique... par exemple si c'est de la musique classique, si c'est du jazz, si c'est du folk, de la musique du monde... »(0), susceptible de former le goût: "L'éducation artistique c'est cette ouverture à pratiquer un choix» (J). La musique est également au service des autres enseignements, comme la lecture : «C'est une façon ludique de rentrer dans certains apprentissages fondamentaux" (J). Mais la musique est aussi envisagée plus globalement pour permettre à l'enfant de découvrir et de développer ses qualités personnelles : "C'est un moyen de les amener à se sentir, à se libérer eux-mêmes, et à prendre conscience de son existence » (A), et sociales : "C'est souvent être vu, être regardé, on s'expose, on se regarde » (J). 
20 Cette courte analyse met en évidence une catégorie d'enseignants qui, bien que se déclarant sans connaissances musicales, ne négligent pas la musique dans leur classe et peuvent se montrer actifs et inventifs dans ce domaine pour proposer à leurs élèves un véritable projet. Ainsi, des enseignants qui apparaissent au départ peu armés peuvent adopter une posture où prédomine la recherche de stratégies, en vue de donner à l'enseignement de la musique un réel statut de discipline d'apprentissage. Nous établissons ici un lien avec le processus de «distanciation " identifié par B. Charlot. Dans ce cas, B. Charlot parle clairement de la production $d^{\prime}$ '« un travail réflexif d'ajustement de la conduite à la situation", ainsi que d'une "régulation" (Charlot, 1999). La réflexion concerne à la fois l'organisation de cet enseignement (par exemple, "le quart d'heure quotidien »), les objectifs ("enrichir ses connaissances", "développer un esprit critique », «la découverte de soi, de l'autre »), les outils (par exemple, « les universels à connaître ", " les chansons populaires au premier trimestre ») et les démarches envisagées.

21 La « régulation » ne se limite pas à la classe et ces enseignants décrivent les stratégies qu'ils adoptent dans leur vie personnelle pour faire évoluer ce qu'ils considèrent comme des compétences limitées. C'est par exemple cette enseignante qui vient juste de débuter la pratique du piano et qui explique : «ce qui a conditionné le fait que je fasse du piano, c'est mon travail » (L). Une autre vient de rejoindre une chorale : «cette année j'ai commencé la chorale » (J). Une autre encore explique que c'est en suivant l'activité musicale de ses deux enfants qu'elle s'est acculturée et que cette quête de musique se poursuit par des échanges réguliers : «j'ai des enfants musiciens et on entretient une culture musicale. Mon fils est étudiant et chaque fois qu'il revient il regarde les disques que j'ai empruntés à la discothèque et il m'en ramène ; il y a une espèce d'échange comme ça » (o).

Qu'en est-il maintenant des enseignants qui ont bénéficié d'une formation et qui déclarent posséder un certain bagage musical puisque certains d'entre-eux se produisent régulièrement en public, voire même ont envisagé à un certain moment de leur vie une carrière musicale?

\section{Quelles pratiques enseignantes pour des professeurs des écoles qui déclarent une solide activité et des connaissances musicales personnelles?}

23 L'analyse des entretiens nous amène à considérer maintenant les enseignants qui ont bénéficié d'une formation musicale au service d'une pratique personnelle importante: "J'ai jamais autant chanté, j'avais l'oreille absolue pendant que j'étais à l'École Normale, tellement on faisait de musique » explique un enseignant qui a un niveau Fin d'Études de conservatoire (D). Il s'agit dans cet échantillon de sujets qui pratiquent la musique et il n'est pas rare qu'ils pratiquent plusieurs instruments: "Je pratique essentiellement l'accordéon diatonique et un peu la guitare " déclare un enseignant qui a bénéficié antérieurement d'une formation en clarinette (B). Un certain nombre d'entre eux se produisent en public régulièrement : "Je joue depuis une vingtaine d'années dans un groupe qui tourne et fait deux prestations dans le mois » $(\mathrm{K})$, ont enregistré : «J'ai déjà enregistré des disques avec des choeurs " (H), ou même caressé l'espoir d'une carrière musicale à un certain moment de leur vie.

Pourtant la description de leurs pratiques enseignantes montre que ces enseignants, qui se déclarent «musiciens », adoptent des attitudes très différentes vis-à-vis de la 
musique dans la classe. Si certains mettent naturellement leurs connaissances musicales au service de leurs élèves, d'autres au contraire limitent leurs interventions dans le domaine.

\section{« Les gens ont envie ou n'ont pas envie » (D)}

En effet, si on trouve dans cette catégorie des enseignants qui développent un enseignement de la musique conséquent : "Ça doit être à peu près une heure par semaine, mais si je sens qu'ils sont dedans, même si c'était pas prévu, je consacre beaucoup de temps » (I), d'autres, au contraire, limitent leurs activités à quelques séances dans l'année: "Normalement le vendredi j'ai une plage, mais c'est tellement passé aux oubliettes depuis quelques mois que je ne sais même plus quand c'est» (K). Pour certains, l'implication dépasse leurs propres élèves puisqu'ils prennent en charge l'Éducation musicale d'autres classes : "Je décloisonne aussi, je fais aussi Éducation musicale avec les $\mathrm{CP}$ » déclare cette enseignante qui anime également la chorale de l'école (C).

Si certains mettent en œuvre des activités variées: «Sur une formule dix minutes, un quart d'heure, voire vingt minutes par jour, une journée est consacrée à l'écoute, une ou deux journées consacrées au rythme et une ou deux journées par semaine sur le chant » (D), d'autres limitent leur enseignement à une seule activité : «En fait enseignement c'est beaucoup dire, c'est très ponctuel, je fais surtout par le chant mais je ne fais pas d'enseignement de la musique » déclare cet enseignant qui ajoute « que la musique à la limite si [il] est trop pressé par un truc, c'est ça qui sautera » (H).

C'est dans cette catégorie que l'on trouve aussi des enseignants qui élargissent les activités musicales aux pratiques créatives, par ailleurs assez rares (Maizières, 2009) : "Après je les mets à plusieurs tout de suite en création, de façon à ce qu'ils commencent à mettre entre eux un dialogue... » (P). La question des outils didactiques est abordée de manière très différente également et paradoxalement, moins l'enseignant fait de musique dans sa classe et plus il a de mal à être précis sur les chansons ou les œuvres étudiées durant l'année : "Chant autour de la montagne, des choses comme ça » résume cet enseignant qui ajoute " on a dû faire deux chansons ou trois quand on est parti en classe de neige, et puis voilà ça se limitait à ça » (K). D'autres, au contraire, ne s'aventurent pas à la légère et s'appuient sur des projets qu'ils décrivent très précisément, par exemple le thème du «Bourgeois gentilhomme revu par Strauss » et qui a fait l'objet d'« une conférence pédagogique comme un petit cours d'approche pour étudier cette musique-là " permettant ensuite à l'enseignante d'étudier avec ses élèves plusieurs versions de ce thème d'époques et de styles différents (I).

De tels propos laissent deviner des finalités très diversement envisagées. À la question des objectifs, certains semblent réfléchir à ce que peut apporter l'activité : «Chanter ensemble... canaliser les gamins... avoir une exigence sur la justesse, sur l'exactitude des rythmes... prendre conscience de sa propre voix... » (H), d'autres, au contraire, citent un certain nombre d'objectifs, à la fois musicaux et transversaux pour chacune des activités. En ce qui concerne le chant, le plaisir est bien sûr visé, mais c'est aussi la justesse, le souffle, la mémoire, l'articulation, etc.,. L'activité d'écoute vise à assurer la formation du goût qui, selon $F$. Brugère, "se présente comme un sentiment qui est aussi jugement" (Brugère, 2000, p. 12) : "Le principe c'est de ramener à décrire ce qu'ils entendent... les instruments, le type de musique, les notions de grave, d'aigu, de rapidité" explique cet enseignant qui poursuit sur la nécessité de donner des repères historiques 
et esthétiques comme "valeurs classiques » afin que ses élèves ne subissent plus "une culture obligatoire » comme "une espèce de rouleau compresseur » (D). La créativité relève également des objectifs : «Je pense que c'est important pour son épanouissement personnel d'être dans une démarche de créativité»(D), dans laquelle "on pousse au-delà de ses possibilités» (C). Et bien sûr, l'éducation artistique c'est le développement de la personnalité : "C'est une manière d'accéder à des savoirs et puis à la liberté, la liberté de savoir, la liberté de partager, de s'ouvrir sur les autres » (I).

Certains enseignants détiennent un savoir musical comme « un objet ou un système qui existe en tant que tel » (charlot, 1999) : "J'avais l'oreille absolue ", «j'ai déjà enregistré des disques", et l'enseigner relève dans ce cas de la posture d'« objectivation ». Leurs objectifs sont clairs et visent des savoirs et des savoir-faire musicaux précis. Leur démarche s'inscrit dans une progression et s'appuie sur des outils clairement identifiés dont ils donnent les titres.

\section{"Autant je me sens bien quand je fais de la musique dans ma vie personnelle, autant à l'école je ne suis pas à l'aise » (B)}

Mais, l'étude des entretiens met aussi en évidence qu'un certain nombre d'enseignants détenant un solide savoir musical personnel, limitent fortement les activités musicales dans leur classe. Il semblerait que certains d'entre-deux parviennent difficilement à transmettre le savoir musical qu'ils ont acquis, ou à s'appuyer sur leurs compétences pour mettre en place un véritable enseignement de la musique. Ils reconnaissent euxmêmes cet écart entre ce qu'ils peuvent faire et ce qu'ils font : "C'est ça qui est terrible, je ne peux même pas dire que j'ai une difficulté à... je ne suis pas dans la position de quelqu'un qui dirait quand je prends un disque j'entends pas ce qu'il faut. Si, là, j'entends tout » $(\mathrm{H})$.

Ces enseignants ont bien conscience de négliger les disciplines artistiques et la musique en particulier et les explications qu'ils donnent illustrent l'aspect social de leur rapport à « l'enseigner » : la lourdeur des programmes : "J'ai l'impression de toujours être en retard dans des choses entre guillemets plus importantes » (K), la pression de l'institution : «Et puis c'est vrai qu'il y a la pression du reste du boulot, les autres collègues qui ne font pas ça... qui prennent de l'avance sur autre chose on se dit qu'on est encore dans les choux, eux ils ont vu les compléments d'objet direct ou les compléments d'objet second moi je ne les ai toujours pas $v u$ » $(\mathrm{H})$, et même de la société : «On a la pression aussi de la société quelque part puisque les parents eux demandent d'avoir des résultats ils leur faut des résultats donc c'est beaucoup maths français » (B).

S'il paraît difficile de s'abstraire de ces contraintes du métier, les propos sur les pratiques déclarées par les enseignants entendus dans le cadre de cette étude mettent aussi en évidence d'autres composantes du rapport au savoir qui pourraient être à l'origine d'un transfert complexe entre les propres compétences de l'enseignant et ses pratiques professionnelles. Une enseignante déclare : «Il y a une interaction ou même un prolongement par rapport à ce que je suis » (I) et une autre dit encore : "On est tellement soimême quand on est enseignant" (F), laissant supposer que c'est davantage encore le rapport «identitaire» au savoir qui pourrait expliquer la difficulté à transposer ses propres connaissances musicales dans la classe. 


\section{Pratiques enseignantes en Éducation musicale et rapport « identitaire » au savoir.}

permet de distinguer deux catégories d'enseignants. Il s'agit, d'une part, d'enseignants qui apparaissent «en règle » avec l'Éducation musicale, soit parce qu'ils ont peu de compétences mais qu'ils font ce qu'ils peuvent pour ne négliger aucune discipline : «J'essaie de m'astreindre à toutes les matières » (E), soit qu'ils se sentent plus à l'aise avec la musique et qu'ils expriment cette aisance à travers leurs pratiques professionnelles. D'autre part, des enseignants reconnaissent qu'ils ont des compétences et pourtant ils n'accordent pas à la musique la place qu'elle devrait occuper dans leur classe : "Je pense qu'il faut que je réaménage mon emploi du temps et que je me botte un peu les fesses aussi, je pense que je suis un peu comme tout le monde quand je ne suis pas assez motivé pour le faire... »(B). On l'a vu, les raisons invoquées dans ce cas relèvent essentiellement de l'extérieur (lourdeur des programmes, pression des collègues, des parents), néanmoins ces enseignants reconnaissent aussi leur implication personnelle. Par exemple, ce dernier parle d'une insuffisance de "motivation ", qu'il explique aussitôt par une certaine appréhension face à des activités qu'il ne maîtrise pas : «Je suis moins à l'aise donc j'appréhende, donc j'ai un peu une stratégie d'évitement... voilà on fait souvent les disciplines dans lesquelles on croit être à l'aise»(B). Il justifie cet "évitement» par la "peur» de ne pas réussir. Ce sentiment de peur est également exprimé par cet autre enseignant qui regrette, malgré ses connaissances, de ne pas faire autant qu'il voudrait, et qui explique qu'il se sent plus à l'aise «avec des choses entre guillemets, dirigées " parce qu'il a "peut-être un petit peu peur de ne pas savoir assez où [il va]» (K).

Par cette dernière remarque l'enseignant exprime clairement son malaise face à une activité pédagogique dont il n'a pas l'entière maîtrise; situation pour le moins insupportable pour celui qui est censé représenter l'« autorité » et le "savoir ». Cette absence de maîtrise ne semble pas toujours consécutive à une insuffisance de connaissances personnelles, mais davantage peut-être au sens attribué à la discipline musicale à l'école. Nous rejoignons sur ce point $G$. Boudinet qui remarque que les conditions qui freinent un certain nombre d'initiatives artistiques dans les classes ne tiennent pas tant au manque de compétences des enseignants qu'à l'incompréhension sur le sens de ces enseignements à l'école (Boudinet, 2007). Et c'est bien cette difficulté à donner du sens à l'Éducation musicale qui est exprimée dans cette déclaration : "Je me sens, j'allais dire, plus animateur entre guillemets qu'enseignant» (K). Quand on sait le sens dévalorisant que peut prendre le terme "animateur» dans le métier de l'enseignement, on comprend la réticence du professeur des écoles à enseigner un domaine dont il ne mesure pas toute la portée éducative. Et d'ailleurs le résultat ne se fait pas attendre puisque la mise en œuvre de certaines activités semble avoir un impact immédiat sur son autorité : «Il y a toujours les gamins qui font les guignols pendant l'écoute c'est vrai que ça pose un problème aussi ça » $(\mathrm{H})$. 

autres et la difficulté pour certains d'entre eux d'imposer la musique comme une véritable discipline d'enseignement, c'est d'abord auprès des parents. Un des enseignants déclare privilégier les connaissances parce que «les connaissances ça se voit... on peut laisser des traces ", ces traces qui semblent nécessaires pour donner une certaine légitimité à son enseignement «par rapport aux parents et par rapport à notre administration » (B). lui-même, c'est aussi à celle qu'il a de lui-même. Ainsi cette enseignante qui se sent «ridicule » quand elle chante dans sa classe explique son appréhension à cause d'une expérience malheureuse vécue alors qu'elle était élève : "Vous chantez faux mademoiselle, allez à votre place. Du coup devant tous les autres, on était une trentaine en quatrième, j'ai rougi et je suis allée m'asseoir et je me suis sentie bête, la plus bête » (N).

En mettant en évidence un certain nombre d'enseignants qui, malgré une solide pratique de la musique, limitent très fortement leur implication dans les activités musicales avec leurs élèves, cette analyse montre l'importance de la composante identitaire du rapport au savoir, tel que la définit Charlot. C'est en essayant de préserver une posture difficile à affirmer dans certains cas que certains enseignants renoncent à ce qu'ils aiment par ailleurs : «Il y a un fossé entre ce que je souhaiterais faire et ce que je fais » (K). En déclarant : "J'ai peut-être un petit peu peur de ne pas savoir assez où je vais » $(\mathrm{K})$, ce même enseignant exprime clairement sa difficulté à comprendre le sens des activités musicales. C'est d'ailleurs pour cette même raison qu'il voit l'intérêt de participer à un concours local dans le cadre des arts visuels : «pour avoir un objectif», sinon il reconnaît que ce serait « comme pour la musique», il en ferait «quand il en aurait le temps» $(\mathrm{K})$.

\section{Conclusion}

L'analyse des entretiens montre que le transfert entre le propre savoir musical de l'enseignant et ses pratiques enseignantes est parfois difficile. Si les pratiques enseignantes en Éducation musicale sont souvent attribuées aux connaissances personnelles de l'enseignant dans le domaine, notre étude montre que ce n'est pas toujours le cas et que les activités musicales mises en œuvre dans les classes ne sont pas toujours proportionnelles aux pratiques et à la formation personnelles des enseignants. Même si on peut toujours supposer un écart entre les pratiques déclarées et les pratiques effectives, il y a fort à penser que les enseignants qui se déclarent «musiciens» ne minimisent pas leurs pratiques de classe quand ils décrivent des activités insuffisantes. Or, c'est bien cette catégorie qui nous interroge plus particulièrement ici. Pour tenter de comprendre les raisons qui amènent certains enseignants à limiter fortement les activités musicales dans leur classe, alors qu'ils ont développé des compétences d'interprétation solides et une appétence pour la musique, nous nous sommes inspirés du modèle d'Escol. Ce modèle nous a permis de mieux comprendre comment le rapport au savoir, et plus particulièrement le rapport «à l'enseigner " permet à des professeurs des écoles d'assumer leur rôle d'enseignant polyvalent avec la charge d'enseigner la musique à leurs élèves.

B. Charlot précise que le rapport au savoir est d'abord un processus impliquant un individu dans toutes les relations qu'il tisse avec «l'apprendre» (1997, p. 67). En ce qui 
concerne l'enseignant, le rapport au savoir en tant que relation à « l'apprendre » est au cœur même de son identité professionnelle, que ce soit par le savoir qu'il est censé transmettre, mais aussi et d'abord le savoir dont il bénéficie pour exercer son métier d'enseignement. En suivant B. Charlot, nous avons analysé le rapport au savoir musical des enseignants interviewés selon trois dimensions: "épistémique " (c'est l'activité que l'individu met en œuvre pour accéder au savoir, et dans notre cas aussi « transmettre » le savoir), « identitaire » (c'est le sens que l'individu accorde au savoir) et « social » (c'est le savoir au cœur de la relation avec les autres).

41 L'analyse des entretiens montre que les enseignants adoptent des stratégies différentes par rapport à l'Éducation musicale, qui peuvent aller de l'«évitement" à une implication régulière et affirmée, en dépit des propres relations que l'enseignant entretient avec la musique dans sa vie personnelle. C'est vraisemblablement la difficulté à appréhender le sens et la valeur de l'Éducation musicale à l'école qui amène certains enseignants à négliger la musique au sein de leur classe alors qu'ils déclarent par ailleurs détenir un sérieux bagage musical. Même avec des connaissances musicales solides, enseigner la musique n'est pas sans risque pour l'enseignant. Certains propos traduisent bien la difficulté pour l'enseignant de garder le contrôle de la classe dans une situation pédagogique insuffisamment maîtrisée. C'est pour eux le risque de voir les élèves adopter des comportements indisciplinés, fragilisant ainsi leur statut de " maître ». En déclarant des difficultés de mise en œuvre en raison d'un environnement social peu favorable à la musique à l'école, ces enseignants tentent ainsi de minimiser leur implication plus personnelle.

À la lumière des analyses que nous avons réalisées et qui montrent une "disciplinarisation" parfois difficile de ses propres savoirs, nous pensons que le développement de la compétence professionnelle, dans le cas de l'éducation artistique, ne peut se faire de façon homogène et encore moins de façon implicite. La formation professionnelle, et aujourd'hui l'université dans le cadre des nouvelles formations au métier d'enseignant, doivent entraîner les professeurs des écoles sur le terrain de la réflexion théorique, afin de leur permettre de questionner le sens et la valeur des disciplines artistiques au sein de l'éducation générale, avant même de donner les outils nécessaires pour répondre aux objectifs de cette éducation.

\section{BIBLIOGRAPHIE}

Alten, M., (1995), La musique dans l'école primaire de Jules Ferry à nos jours, Issy-Les-Moulineaux :

Éditions E.A.P.

Baillat, G., Espinoza, O., Vincent, J., (2001). De la polyvalence formelle à la polyvalence réelle : une enquête nationale sur la pratique des enseignants du $1^{\mathrm{er}}$ degré. Revue française de pédagogie, $n^{\circ} 134,123-136$.

Bautier, E., Rochex, J.-Y., (1998), L'expérience scolaire des nouveaux lycéens. Démocratisation ou massification? Paris : Armand Colin. 
Boudinet, G., (2007), Enseigner la musique aujourd'hui : Oui, mais pourquoi ? Conférence Cité de la Villette, janvier.

Brugère, F., (2000), Le goût. Art, passions et société, Paris : PUF.

Charlot, B., Bautier, E., Rochex, J.-Y., (1992). École et savoirs dans les banlieues... et ailleurs, Paris : Armand Colin.

Charlot, B., (1997), Du rapport au savoir. Éléments pour une théorie, Paris : Anthropos.

Charlot, B., (1999). Le rapport au savoir, Éducation et formation, in J. Bourdon \& C. Thélot (Dir.), L'apport de la recherche aux politiques éducatives, Paris : CNRS Éditions, 17-34.

Jahier, S., (2006). L'éducation musicale à l'école : les pratiques pédagogiques et le rapport au savoir musical des enseignants du primaire. Thèse de doctorat, Université de Paris 10-Nanterre.

Maizières, F., Vilatte, J.-C., Dupuis, P.-A., (2007). Pratique de la musique en amateur des enseignants du premier degré et enseignement de la musique, Communication au Congrès AREF, Strasbourg, Août.

Maizières, F., (2009). Le rapport à la musique des enseignants du premier degré : rapport personnel, rapport professionnel. Thèse de doctorat, Université Nancy 2.

France : ministère de l'Éducation nationale (2002). Horaires et programmes d'enseignement de l'école primaire, B.O., $\mathrm{N}^{\circ} 1,14$ février, 1-100.

France : ministère de la Jeunesse de l'éducation et de la recherche (2003). L'Éducation aux Arts et à la Culture (Rapport de l'Inspection Générale présenté par C. Juppé-Leblond, A. Chiffert, G. Lesage \& M.-M. Krynen, Janvier).

France : ministère de l'Éducation nationale de l'enseignement supérieur et de la recherche (2007). La mise en œuvre de l'éducation artistique et culturelle dans l'enseignement primaire (Rapport l'Inspection générale - nº 2007 - 047 présenté par V. Buysse, V. Maestracci, J.-Y. Moirin \& C. Saint-Marc, Mai).

Piriot, M., (1997). Pratiques culturelles chez les instituteurs et réinvestissement pédagogique. Thèse de doctorat, Université Paris 5-René Descartes.

Rochex, J.-Y., (1995), Le sens de l'expérience scolaire : entre activité et subjectivité, Paris : PUF.

Suchaut, B., (2000), La musique à l'école primaire. Analyse des pratiques enseignantes. Iredu-CNRS et Université de Bourgogne.

\section{NOTES}

1. B. Suchaut cite notamment le travail effectué par une équipe de conseillers pédagogiques (Musique au quotidien, CRDP de Dijon, 1996), qui reste une référence incontournable encore aujourd'hui, ainsi que différentes études auxquelles il a contribué (Mingat, A., Suchaut, B., (1994), Évaluation d'une expérimentation d'activités musicales en grande section de maternelle, Cahier de l'IREDU, $N^{\circ} 56$, décembre; Mingat, A., Suchaut, B., (1996), Incidences des activités musicales en grande section de maternelle sur les apprentissages au cours préparatoire. Les sciences de l'éducation pour l'ère nouvelle, Vol. 29, $\left.\mathrm{N}^{\circ} 3,49-76.\right)$

2. Éducation, Socialisation et Collectivités Locales (département de Sciences de l'éducation, Université Paris 8 Saint-Denis) 
3. Précisons que les programmes en vigueur au moment de l'enquête sont ceux de 2002. L'éducation artistique regroupe alors les Arts visuels et l'Éducation musicale pour un volume horaire hebdomadaire de 3 heures. L'Éducation musicale s'appuie sur la pratique du chant (interprétation d'une dizaine de chansons), l'écoute (reconnaissance des éléments caractéristiques d'une œuvre), les pratiques instrumentales (recherche et invention à partir d'instruments à percussion et d'objets sonores) et la réalisation de projets musicaux, qui visent le réinvestissement des acquis des autres activités pouvant donner lieu à des pratiques de création musicale (B.O. $N^{\circ} 1,14$ février 2002).

4. IME : Institut Médico-Educatif. Désigne un établissement scolaire qui accueille des élèves déficients

\section{RÉSUMÉS}

La musique à l'école primaire dans le système français a de tout temps été considérée comme le "parent pauvre» de l'éducation pour des raisons institutionnelles, sociales, mais aussi personnelles à l'enseignant qui se considère souvent peu armé pour l'enseigner. Pourtant, une étude montre que, contrairement à l'idée communément admise, les enseignants qui pratiquent la musique ou qui ont bénéficié d'une formation personnelle ne mettent pas forcément en œuvre davantage d'activités musicales dans leur classe (Maizières, 2009). Analysé à partir des outils socio-linguistiques d'Escol, les propos d'un certain nombre d'enseignants (16) confirment que le transfert entre leurs propres savoirs musicaux et leurs pratiques enseignantes est parfois complexe.

Music at primary school level has always been considered as the "poor relation" of education for institutional, social reasons, but also because of the teacher's personal reasons. Indeed he often considers himself not trained enought teach it. Yet, a study shows that, contrary to the commonly thought idea, the teachers who practice music or who benefited from a personnal training, do not put necessarily implement more musical activities in their class (Maizieres, 2009). Analysed from the social-linguistic tools of Escol, the remarks of a certain number of teachers confirm that the transfer between the teachers'own musical knowledge and their teaching practices is sometimes complex.

\section{INDEX}

Mots-clés : éducation musicale, pratiques enseignantes, professeurs des écoles, rapport au savoir

Keywords : musical education, primary school teachers, relationship to knowledge, teachers pratices 
AUTEUR

FRÉDÉRIC MAIZIÈRES

(IUFM Midi-Pyrénées, Université Nancy 2) 